\title{
Correction to: Innate Immune Signaling and Alcohol Use Disorders
}

Leon G. Coleman, Jr. and Fulton T. Crews

\section{Correction to: \\ Chapter "Innate Immune Signaling and Alcohol Use Disorders" in: L. G. Coleman, Jr. and F. T. Crews, Handbook of Experimental Pharmacology, https://doi.org/10.1007/164_2018_92}

In the second paragraph of section 1.3 on line 27 , the text appears incorrect as glutamatergic and neurons. It should read as glutamatergic and GABAergic neurons. The original chapter was corrected. 